\title{
Behavioral responses of losiderma serricorne and chemical composition of essential oil isolated from nicotiana tabacum leaves*
}

\author{
Li-Hong Tao, Yuan-Mei Chen, Ting-Ting Cai and Min Ye† \\ State Key Laboratory for Conservation and \\ Utilization of Bio-Resources in Yunnan \\ Yunnan Agricultural University, \\ Kunming, Yunnan, 650201, China \\ $\dagger$ E-mail address: yeminpc@126.com \\ Wen-Neng Li, Sen Ren and Bo Zhang \\ Hongyun Honghe tobacco (Group) Co., \\ Ltd. Honghe Cigarette Factory, \\ Mile, Yunnan, 652300, China
}

\begin{abstract}
Essential oils can have an impact on behavioural responses of cigarette beetle (Lasioderma serricorne). In this study, we provide evidence that some chemical compounds in essential oil of tobacco (Nicotiana tabacum) leaves, which were subjected to hydrodistillation and extracted with n-hexane, can strongly attract $L$. serricorne significantly. Two main chemical constituents, 6, 8-nonadien-2-one, 8-ethyl-5 (1-methylethyl) and 1-(2, 6, 6-trimethyl-1, 3-cyclohexadien-1-yl) 2 -buten-1- one, in the essential oils were confirmed by GC-MS analysis. This result could be helpful to find some chemical compositions from $N$. tabacum as leading compounds for development of new bionic attractant.
\end{abstract}

Keywords: Lasioderma serricorne, Nicotiana tabacum, behavioural responses, essential oil, GC-MS

\section{Introduction}

Tobacco, Nicotiana tabacum, originated in South America. It is mainly distributed in South America, South Asia and China [1]. During storage, tobacco vulnerable to tobacco beetles infestation. Tobacco beetles leaving smell or holes in the cigarette and serious impact availability and quality of tobacco [2].

The cigarette beetles, Lasioderma serricorne, is one of the most serious pest of stored tobacco, tobacco products, cereal grains and processed foods and cause

\footnotetext{
${ }^{*}$ This research was supported by the Science and Technology Project of Hongyun Honghe Tobacco (Group) Co. (Grant No. HYHH2012YL05) and the National Natural Science Foundation of China (Grant No. 31460487).
} 
significant damage to the multibillion dollar food and tobacco industries worldwide each year[3-6]. L. serricorne is a pervasive pest with an international distribution mainly in tropical, subtropical and warm-temperate climates [7].

In this paper, we studied the attracting activity of essential oils from $N$. tabacum L. leaves on L. serricorne, and tried to find some chemical compositions from $N$. tabacum as leading compounds for development of new bionic attractant.

\section{Materials and Methods}

\subsection{Plant materials}

The dried leaves of N. tabacum were obtained from Hongyun Honghe tobacco (Group) Co., Ltd. Honghe Cigarette Factory, Mile, Yunnan Province and then kept in a dark and cold condition until used shortly after that for the experiments.

\subsection{Insects}

The cigarette beetles ( $L$. serricorne) were obtained from laboratory cultures maintained for the last 2 years in the dark incubators at $28 \pm 0.5^{\circ} \mathrm{C}$ and $70 \pm 0.5 \%$ relative humidity. Unsexed adult beetles used in all the experiments were about 10 days old.

\subsection{Extraction of essential oil}

The powder of $N$. tabacum Leaves was subjected to hydro distillation using a modified Clevenger-type apparatus for $4 \mathrm{~h}$ and extracted with $n$-hexane. Anhydrous sodium sulphate was used to remove water after extraction. The obtained essential oil was kept in sealed glass vial at $4{ }^{\circ} \mathrm{C}$ until further use.

\subsection{Behavioral responses test}

Behavioral responses of the cigarette beetles (L. serricorne) were investigated with a glass $Y$-tube olfactometer of $1 \mathrm{~cm}$ internal diameter, with a $14 \mathrm{~cm}$ stem and $15 \mathrm{~cm}$ arms at a $75^{\circ}$ inner angle. Air was pumped through an activated charcoal filter and a humidifier through Teflon tubing and divided by a glass Y-junction. The two airflows then passed through two separate flow meters, which regulated the flow rate to $500 \mathrm{~mL} \cdot \mathrm{min}^{-1}$. The air then passed into two spherical glass vessels (i.d. $35 \mathrm{~mm}$ ), into which the essential oil was placed. From here, the air from the vessels flowed into the arms of the olfactometer. The essential oil of $N$. tabacum. Was diluted with $n$-hexane at two different concentration levels of $2.5 \mu \mathrm{g} \cdot \mu \mathrm{L}^{-1}$ and $5.0 \mu \mathrm{g} \cdot \mu \mathrm{L}^{-1}$, respectively. Ten microlitres 
of essential oil diluents was added to a filter paper (diameter $3.0 \mathrm{~cm}$ ). The solvent was allowed to evaporate for $30 \mathrm{~s}$ before the filter paper put into the odor vessel of olfactometer. $N$-hexane was used as control.

The Unsexed adult beetles about 10 days old were used for the tests. The beetles were individually introduced into the olfactometer. The beetles in the olfactometer chose one side of the two arms by chemotaxis at the Y-junction of the olfactometer. The observation ended when the beetles reached the finish line (3.5 $\mathrm{cm}$ from Y-junction) of one of the olfactometer arms, with maximum observation duration of $5 \mathrm{~min}$ per beetle. The treatment and control arms were reversed after half of the beetle tests had been accomplished. Experiments were conducted in a climate-controlled room $\left(26 \pm 1{ }^{\circ} \mathrm{C}, 70 \pm 5 \% \mathrm{RH}\right)$ under one 40-W fluorescent lamps, fitted with a prismatic filter, to ensure a completely even distribution of light. This illuminated the Y-tube with a light intensity of 750 lux (measured by a digital luxmeter, model 7001, Germany). Ten different beetles were used for each replication and five replications were carried out for behavioral responses test. Between trials, all glassware was washed with acetone and distilled water and then baked at $210^{\circ} \mathrm{C}$ over night to remove any volatiles adhering to the glass.

\subsection{Identification of essential oil}

Essential oils were identified by gas chromatograph-mass spectrometry (GC-MS). Gas chromatographic analysis was conducted on a gas chromatograph (Agilent 7890, America) coupled to a quadrupole mass spectrometer (Agilent $5975 \mathrm{~N}$, America). Separation of compounds was performed on a HP-5MS capillary column (J\&W Scientific, $30 \mathrm{~m} \times 0.25 \mathrm{~mm}$ i.d., film thickness $0.25 \mu \mathrm{m}$ ). The GC oven temperature was programmed as follows: from 40 to $250^{\circ} \mathrm{C}$ at a rate of $5^{\circ} \mathrm{C} \mathrm{min}-1$ and held for $10 \mathrm{~min}$. The injector temperature was $260^{\circ} \mathrm{C}$. Helium of high-purity was used as the carrier gas at a flow rate of $1.0 \mathrm{~mL} \cdot \mathrm{min}^{-1}$. Split injection mode was used and split ratio was 10:1.

The mass spectrometer was fitted with an EI source operated at $70 \mathrm{eV}$ with a source temperature of $230^{\circ} \mathrm{C}$, and mass spectra were recorded in the range of $\mathrm{m} / \mathrm{z}$ 50-550 amu in the full scan acquisition mode. The quadrupole temperature was $150^{\circ} \mathrm{C}$, and the interface temperature was $280^{\circ} \mathrm{C}$. Essential oils were identified by comparing the obtained mass spectra of the analytes with those of authentic standards from the NIST 08 library with a resemblance percentage above the ninety percent. The chemical components relative content was calculated based on the peak area of total ion current chromatogram. 


\subsection{Statistical analysis}

Chi-square tests were used to test the hypothesis that the numbers distribution of side-arm choices between the essential oil and control. Independent-Sample T tests were used to determine significant differences between the mean times of individual beetle making a choice between the essential oil and control. All the data were analyzed with statistical package SPSS 17.0

\section{Result}

\subsection{Responses of L. serricorne to the essential oil}

The results obtained on the olfactory response of $L$. serricorne between different doses of the essential oil and blank control were showed in figure 1.

Figure 1 clearly illustrated that the beetles (L. serricorne) was attracted to essential oil from tobacco leaves significantly $(p<0.01)$ when a dose of the essential oil was less than $500 \mu \mathrm{g}$. But when a dose of the essential oil was 1000 $\mu \mathrm{g}$ the beetles (L. serricorne) did respond significantly better to blank control.
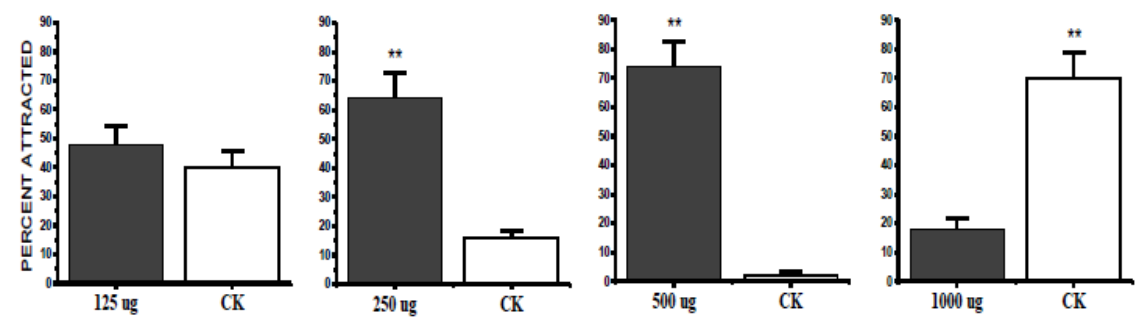

Fig. 1. Mean \pm SD percentages of cigarette beetles in an olfactometer that moved upwind toward the essential oil or blank control. An asterisk above bars indicates they are significantly different by a paired $t$-test at $P<0.01$.

\subsection{GC-MS analysis of the essential oil}

Total ion current (TIC) chromatogram of mass spectrometry for essential oil was showed in figure 2 .

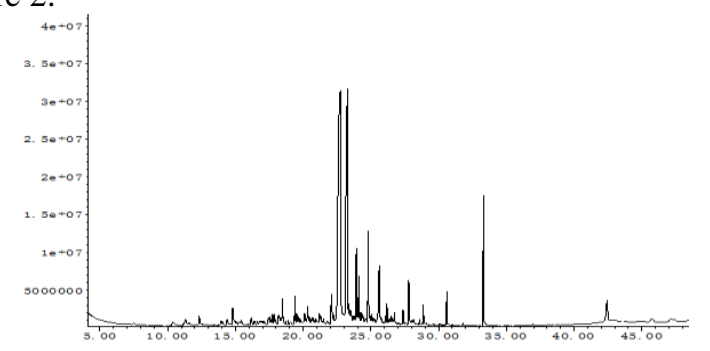

Fig. 2. TIC of essential oil from leaves of N. tabacum. 
Based on the total ion current chromatogram of mass spectrometry, there were tow main chemical constituents in the essential oil. They were 6, 8-Nonadien-2- one, 8-methyl-5-(1-methylethyl) (retention time $22.763 \mathrm{~min}$ ) and 1-(2, 6, 6-Trimethyl-1, 3-cyclohexadien-1-yl)-2-buten-1-one (retention time $23.256 \mathrm{~min}$ ). Result of quantitative analysis by peak area normalization showed that content of 6,8-Nonadien -2-one,8-methyl-5-(1-methylethyl) and 1-(2,6,6-Trimethyl-1, 3-cyclohexadien-1-yl)- 2-buten-1-one in essential oils was up to $31.748 \%$ and $18.009 \%$, respectively.

\section{Discussion}

L. serricorne is major pests on a wide variety of dry and durable stored agricultural products [8]. There are many different traps baits of L. serricorne were reported to attract tobacco beetles, such as sex pheromone serricornin, red chilli powder, chilli volatiles, combining pheromone with plant-derived volatiles and other synthetic sex pheromone [5, 8-11]. The essential oil was extracted by Soxhlet method from Ailanthus altissima Swingle bark could repelled and fumigate L. serricorne adults [12]. Essential oils isolated from Cymbopogon citratus and Eucalyptus citriodora could repel Tribolium castaneum [13].

The data from this study provide evidence that essential oils from tobacco leaves can attract $L$. serricorne significantly. It is similar to $L$. serricorne attracted by chilli volatiles [8]. But there are differences between tobacco essential oils and chilli volatiles, there are come from host plant and another plant, respectively. If we combine tobacco essential oils with chilli volatiles instead of sex pheromone, it not only enables build a green tobacco industry but also dramatic save prevention costs.

There are Two main chemical constituents, 6, 8-nonadien-2-one, 8-ethyl-5 (1-methylethyl) and 1-(2,6,6-Trimethyl-1,3-cyclohexadien-1-yl)2-buten-1-one, in the tobacco essential oils were confirmed by GC-MS analysis. They may be two of the food pheromones of tobacco beetles. According to the report, there are many different pheromones consisting of essential oil components of various plants and released by insects and arachnids (pests) [14]. Therefore, strategies for pest management by using pheromones seem to be cost-effective, and environment friendly compared to the use of insecticides.

\section{References}

1. Flora of China. 1978, 61(1): 152.

2. Ryan L. Post harvest infestation control. London: Chapman \& Hall, 1995.

3. P. Kaelina, L. Zauggb, A. M. Albertini, et al. Activity of Bacillus thuringiensis isolates on Lasioderma serricorne (F.). Journal of Stored Products Research, 1999, 35: 145-158. 
4. S. C. Papadopoulou, C.G. Athanassiou. Lariophagus distinguendus (F.), an ectoparasitoid of Lasioderma serricorne (F.), found for the first time in tobacco stores in Greece. Journal of Pest Science, 2004, 77: 183-184.

5. R. M. Mahroof, T. W. Phillips. Mating disruption of Lasioderma serricorne in stored product habitats using the synthetic pheromone serricornin. Journal of Applied Entomology. 2014, 138: 378-386.

6. RM Mahroof, TW Phillips. Life history parameters of the cigarette beetle, Lasioderma serricorne (F.) as influenced by food resources. Journal of Stored Products Research. 2008, 44: 219-226.

7. PA Edde, M Eaton, SA Kells, et al. Biology, behavior and ecology of pests in other durable commodities. In: Stored product protection. Kansas State University Press, 2012, 45-61.

8. R. M. Mahroof, T. W. Phillips. Responses of stored-product Anobiidae to pheromone luresand plant-derived volatiles. Journal of Applied Entomology. 2008, 132: 161-167.

9. W. A. Jonfia-Essien, P. G. Alderson. G. Tucker, et al. Behavioural responses of Tribolium castaneum (Herbst) to volatiles identified from dry cocoa beans. Pakistan Journal of Biological Sciences. 2007, 10(20): 3549-3556.

10. M. Fardisi, L.J. Mason. Influence of lure (food/sexpheromone) on young mated cigarette beetle (Lasioderma serricorne (F.)) flight initiation. Journal of Stored Products Research. 2013, 53: 15-18.

11. L. L. Li, S. C. Zhang, X. L. Liang, et al. Effects of several factors on trapping quantity of Grapholitha molesta busck by sex pheromone. Plant Diseases and Pests 2012, 3(5): 8-10.

12. J. H. Lv, Y.L. Shi. The Bioactivitiy of essential oil from Ailanthus altissima Swingle bark on Lasioderma serricorne (F.)First International Conference on Cellular, Molecular Biology, Biophysics and Bioengineering (CMBB). 2010 .

13. O. V. Jesus, S. N. Luz, E. E. Stashenkob. Bioactivity against Tribolium castaneum Herbst of Cymbopogon citratus and Eucalyptus citriodora essential oils grown in Colombia. Pest Management Science.2010, 66: 664-668.

14. M. L. Marianne, B. B. Gerhard. Essential oil components as pheromones. Flavour and Fragrance Journal. 2011, 26: 357-377. 